\title{
Integra Dermal Regeneration Template
}

National Cancer Institute

\section{Source}

National Cancer Institute. Integra Dermal Regeneration Template. NCI Thesaurus. Code C137997.

A proprietary bilayer membrane system for skin replacement. It is composed of a dermal replacement layer made of a porous matrix of cross-linked bovine tendon collagen and glycosaminoglycan manufactured with a controlled porosity and defined degradation rate, and an epidermal substitute layer made of polysiloxane silicone to control moisture loss from the wound. 